\title{
SOCIETY: A Social Reading Application to Join Education and Social Network Experience
}

\author{
Elena Guercio ${ }^{1}$, Fabio Luciano Mondin ${ }^{1}$, Maurizio Belluati ${ }^{1}$, and Lucia Longo ${ }^{2}$ \\ ${ }^{1}$ Telecom Italia S.p.A. Torino, Italy \\ \{elena.guercio, fabioluciano, maurizio.belluati\}@telecomitalia.it \\ ${ }^{2}$ Politecnico di Torino \\ lucia.longo@polito.it
}

\begin{abstract}
This paper describes a social reading application designed for education and school communities based on electronic book reading experience. Both design and evaluation followed a user centered approach in order to obtain a custom made application called SOCIETY, to be used by community based groups with similar reading interests, running both on IOS and Android System. A special emphasis was given on personal and social aspects in writing and sharing notes with the community groups and the "facebook or twitter" large community to better understand how that can improve the learning process.

Beside showing a high interest on society@ school app our trial evidenced the need to separate school community from wider social networks, a need which seems to be important for students. That stresses the importance of privacy management in school applications design, because students seem to be worried about sharing information and mixing school and social networks contexts.
\end{abstract}

Keywords: Social reading, privacy/respecting, User Experience, School, User Interface, Personal, Social.

\section{Introduction}

The number of social network users is expected to grow in the next few years. According to a research performed by eMarketer [1] in 2011 there were 1.20 billion people using social networks, number which is expected to grow to 1,85 Billion through 2014. Social networks are making multimedia content sharing very easy and funny, also due to the possibility of exchanging opinions and thoughts together with digital content. On the content side, expenses related to digital content are probably going to grow as well as delivery and access to digital content becomes easier and easier. The $2011 \mathrm{PwC}$ report [2] forecasted consumer spending for entertainment and media industry to surge from $\$ 1.4$ trillion in 2010 to $\$ 1.9$ trillion by 2015 . Digital content certainly include e-books, and in particular Children and Young Adult's ebooks, which according to the American Association of Publisher showed a massive +475.1\% increase from 2011 to 2012 [3]. E-book adds a new dimension to traditional books: interactivity. E-Book enables users interaction both with the media and between users, creating and sharing notes and content on top of the e-book content. 
Market data and technical innovation aspects seems to open interesting opportunities in the education field, and that is the main reason standing behind the choice of developing SOCIETY (SOCIal Ebook communiTY) application: giving students the possibility to add a note reporting their opinion, summary, or link to external content, making the e-book 'live' and dynamic. A note may contain multimedia content (i.e. audio, video, image), extending the original plain text book as a real and new multimedia dimension.

An application to be used by students should be designed in order to be suitable for this scenario, finding a proper trade-off between a "natural" wider use of social networks and privacy concerns. Sharing content, especially in the education environment, implies also the definition of instruments and rules for identifying "who is sharing what and with whom' and for moderating the shared extra contents in order to avoid misleading or offensive notes.

Moreover the integration with general social networks (i.e. Twitter, Facebook), should be addressed carefully. Giving right to all user profile information just for posting a message and sharing it on user preferred social network, could be enough for reject the authorization to proceed.

\section{State of the Art}

\subsection{Technical View}

As it was said previously the e-book is more than a different way to read: it is a new interactive experience. Reading a book on a tablet gives the user the possibility to perform actions on the book, spreading from basic operations such as changing font style or size, to advanced operations such as adding notes and multimedia content to the original book.

The most popular reading applications, Apple iBook and Amazon Kindle, someway give the user such kind of interactivity. The user can underline text or add textual notes and save them on the cloud in order to find them among their different terminals, but it's not possible to interact with people reading the same book except for the possibility to post a note as a new status on the chosen social network (i.e. Twitter or Facebook). Other applications such as Readmill, Inkling and Kobo extends the possibility to share text annotations among users owning the same e-book and discuss by using comments.

Adding text notes to e-books is anyway just a small part of a wider story. Content could be extended by adding multimedia annotations by giving the users the possibility to add audio, photo and video to be enjoyed using the application itself.

On the other hand, sharing multimedia content requires keen user identification in order to avoid privacy and moderation issues. Teachers should be able to delete inappropriate contents or promote the best contributions as new teaching material for the entire class.

Moreover, a social reading solution should be able to manage content protected by Digital Right Management system in order to make it work also with bestsellers and editorial books. 


\subsection{User Experience View}

As it was also explained previously, compared to traditional paper books, eBooks have several advantages [4]. Digital books can give the possibility to overcome some reading diseases by means of customizable displays, page and font layout changes or by combining text reading with auditory stimuli. E-books are also easy to be transported and always available. According to Abram, these features can revitalize the Past, with a sort of culture and thinking restoration, by re-inventing books in a creative way, through video, animations and sounds.

In the article "Mobile phones in the Classroom: If You Can't Beat Them, Join Them', Scornavacca, Huff And Marshall [5], by collecting data about 1200 students in a large undergraduate class, showed "that both students and instructors can benefit from an additional channel of communication - SMS messages via mobile phones - in the classroom. The lecturer perceived a gain of quality and quantity of feedback from the students. Students indicated that the system was useful - making classes more interesting and interactive".

It was 2002 when Takeda e Suthers [6] tried to create a social reading web tool (Pink), while in $2008 \mathrm{Kim}$, Farzan and Brusilovsky [7] evaluated an interface in a classroom study, designed to allow the users to identify spatial regions in textbook pages and use them for bookmarking, commenting, and sharing opinions with others. This work stated that "annotation is a natural and beneficial activity for users because it assists them in reading, writing, sense-making, indexing and retrieving". Both works showed how users seem to need online tools to support discussions and learning by making artifacts, references and annotations in a easily way .

Other researchers, such as Kiili et al. [8] tried to identify different profiles for collaborative reading in order to find out different styles in sharing content and found five different profiles: co-constructers, collaborators, blenders, individually oriented readers, and silent readers.

This is the context in which Society try to take its place. Our prototype is based on the idea that reading can be a social and shared experience and annotations help the reader in text understanding, storage and organization and the reading experience will be made more and more interactive and significant on mobile devices such as tablet PC.

\section{Application Description}

With SOCIETY (Fig.1) users can share their reading experience with other readers or books' authors. For instance in educational environment they can share their learning experience with classmates and teachers by creating and commenting annotations on schoolbooks both from home and from school, experiencing a new and more dynamic way of collaborative teaching and learning. The design of SOCIETY kept the focus on allowing the re-use of well-known interaction processes such as notifications, text selection, to build a new interaction process in App by "recycling" the previous know how and by using it in a simple and quick way. So that users can choose 

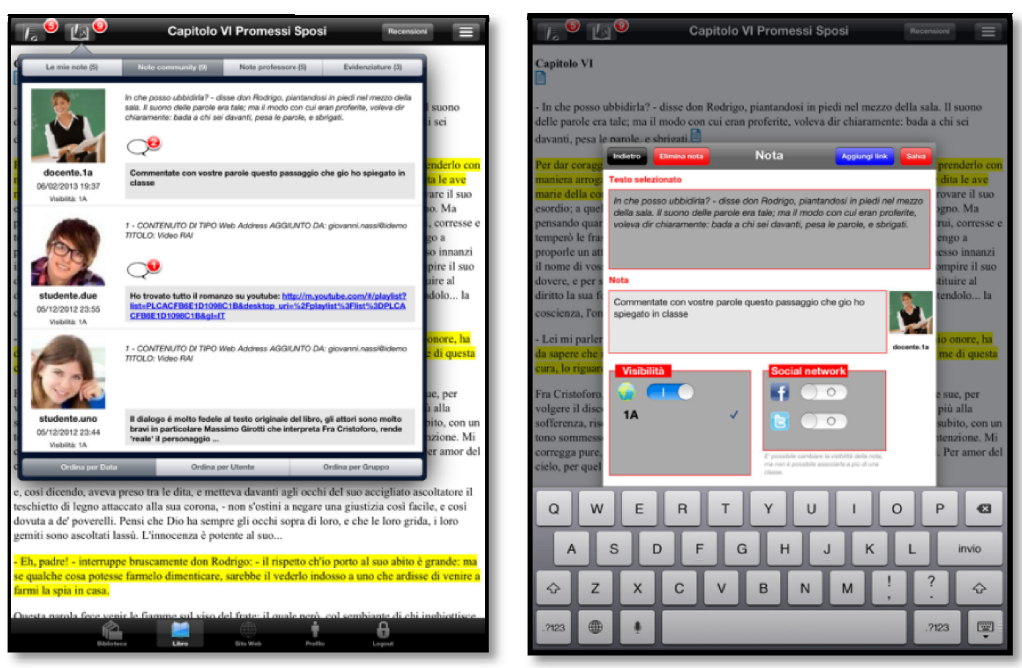

Fig. 1. Application screenshots

the right level of visibility of their annotation, decide if they have to be private, shared only with the author, within a group, or within the whole community and larger social network (i.e. facebook or twitter) too.

SOCIETY was then developed as a prototype App, keeping the user at the center of the design process, and once developed it was tested with final users.

In designing SOCIETY the attention was kept on the actions performed by users using a paper book trying to rebuild this user experience in a simpler, digital way: then, using SOCIETY, the user does not only read e-books, he also underlines text, put notes beside the text, he sometimes places bookmarks, keeps it, lends it, talks about it with his friends.

\section{$4 \quad$ User Requirements and Field Trial}

In general e-books make learning easier through collaboration. The students often prefer giving their comments to a text by writing them due to "social" implications of speaking with their teachers and classmates.

In designing Society@school app we had to take into account both data coming from trials and literature. We also involved some teachers and the Director of an high school in Trento, through some open face to face interviews.

The first challenge, was to combine basic requirements (text customization, annotation sharing, access from smartphone and web, etc) with some more specific requirements, such as having separate views for private and public notes was highlight.

The extension of school contents towards large communities (facebook and twitter overall) seemed to be a very promising opportunity as "everyday students usually study and do their homework with Facebook open”. Social Network sharing could be 
important in order to create a link between the students' school and private life, stimulating them this way to write and share contents about school texts or reading books.

The App was developed both for IOS and Android with the perspective to reach as many device as possible, keeping anyway the same approach to logical design of GUI information, accessibility, navigation, number and types of functionalities and trying to give a common look and feel to both apps.

Once developed, the app was tested by means of a six- months field in Trento, in the same school in which we collected most of the requirements.

The users' sample involved 42 students in two different classes and 3 teachers. The trial started with s short presentation of the App to the students and teachers both to explain the App focal points and usages, and to support the users with the installation on their devices.

Afterwards, we constantly monitored the app usage in different contexts with different teachers, trying to collect as many information as possible from the anonymous log files, but also getting explicit and direct opinions or comments through a specific in-app feedback functionality. Finally we used a poll to get a general opinion about the whole trial and the App's functionalities and performances. In order to stimulate feedbacks a specific web portal section was developed for the description of the development team actions related to users' feedback.

The data collected during the field trial could be classified as:

- spontaneous input and suggestions from the students during the App interaction by email and a specific App software button

- students log files (access number, more clicked functionalities, etc...)

- group interviews (about 10 in 2 classes) with students (Fig. 2)

- 3 face to face interviews with teachers (Fig. 2)

- interviews with students' parents (6)

- final questionnaire
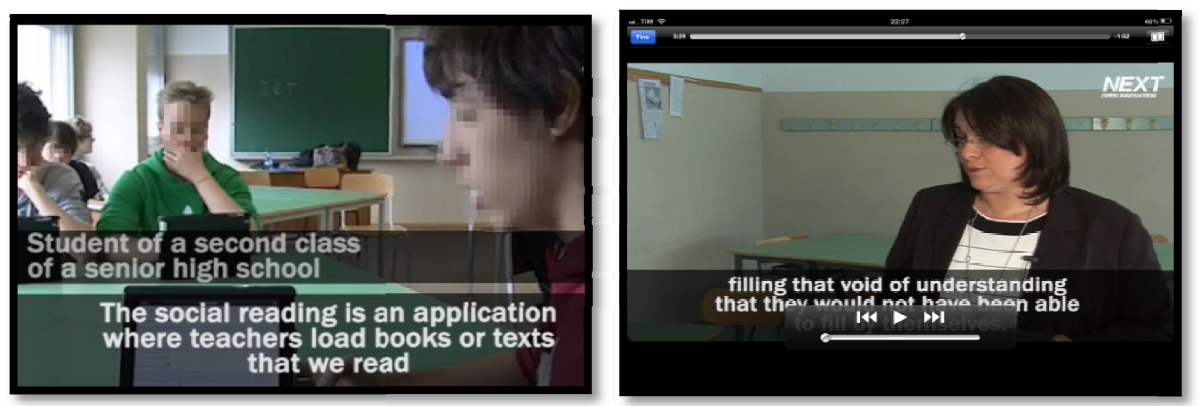

Fig. 2. Face to face interviews with students (on the left) and teachers (on the right)

We used this information to evaluate the usefulness of the App in the school context, but also to optimize and re-design the App and to make it users-tailored. A lot of interesting suggestions were collected, the most important were: 
- Notes were not enough: comments over notes were introduced

- explicit highlighting of the notes' cited text.

- Number of notes and comments shown in library section of the app.

- Visibility of notes on class basis.

Unexpectedly "sharing activities to the large communities of Facebook and Twitter" (i.e. not default sharing of notes towards community) from a student perspective, should be kept apart from the friends community making them two worlds to be at least interconnected but not tied.

Students asked for the same social approach and functionalities of their preferred social network, but they adopted a different behavior during the trial. They used to share school data and experience just inside the class and use Facebook and Twitter for extra scholastic experiences: independently from the fact that they received a positive mark from the teacher or even if a note had a lot of comments from the classmates.

We also offered the student a "sign up" functionality via Google, Facebook and Twitter, in order to simplify the registration process, but in nearly every case students weren't interested in using it.

User were concerned about the number and nature of grants and permission to give to the app on Facebook or Twitter. It's important to ask for the minimal and necessary grants in merging the personal and school "environments". Asking access to the entire user profile information just for posting a single message (i.e. user status) seem to be a strong concern for the users and should then be avoided. It's necessary instead to ask for the minimum possible number of grants and explain well why you are asking for it.

To make clear this design choice for the user, we put the default to the direct connection to Facebook and Twitter "off".

In general the survey showed a satisfactory experience (Fig.3) with Society@school: the "vote" of the app was 7.3 on 1 to 10 scale. App and the shared suggestions and optimizations on App proposed both by teachers and pupils were taken into account in the new App version.
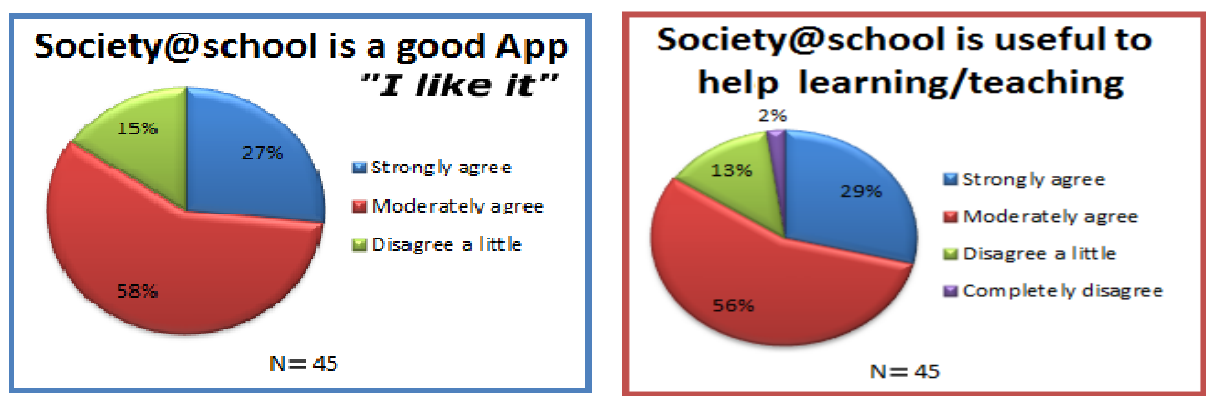

Fig. 3. Users' comments on Society@school App 


\section{Conclusions and Next Steps}

Our trial results show us how high has been the interest on society@ school app, as the figure n. 4 shows (Fig. 4).

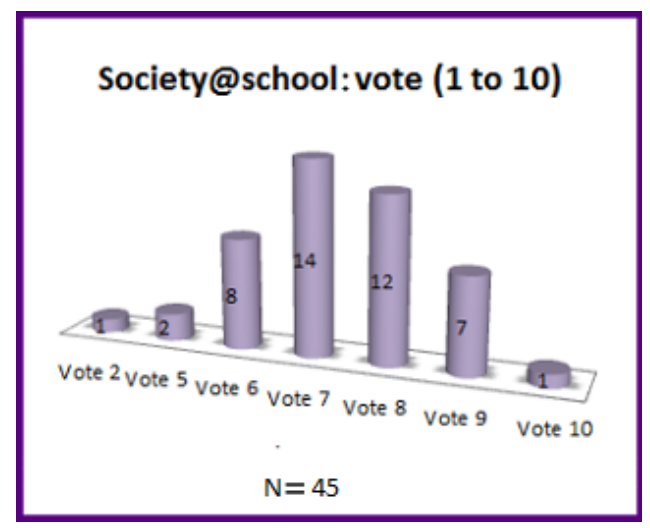

Fig. 4. Final evaluation of society@ school app

Over $85 \%$ of users have expressed a positive opinion on it, highlighting the added value to the traditional learning. Trialists have also judged the app very user friendly and easy to use. In fact, in the interviewed sample, $82 \%$ of them have evaluated easy to read notes, $71 \%$ easy to share them, and still $71 \%$ easy to insert new one or edit it.

Very important is the "discovery" that, more for students than for teachers, a separation between school community and the wider social networks community is necessary. Thanks to the log files we traced, we have found that only a few percentage of users (about 10-15\%) has linked own society@school account to the Facebook or Twitter profiles. Moreover, even among those who have linked society@school profile to Facebook or Twitter, few users have posted their own society@school activities. This finding suggests how important is the privacy management in school applications design, because people, especially teenagers, seem to be very cautious and worried about sharing information and mixing school and social networks contexts.

Our trial has been also very useful to optimize our app, adding new features requested by users such as the opportunity to comment others' notes or to highlight text you are reading. Then about privacy, the "shrewdness" we adopted:

- the new inserted note has to be checked by the student before be shared with others (instead of that the note is just "private")

- then, in a second step, student can decide if sharing or not his note on Facebook and Twitter

The next step of this experimentation is to extend the trial to other primary and secondary schools (Bolzano, Bologna and Caltanissetta are starting at the time of writing), to other classes in the same school in Trento and also to some university courses and community environments. 
The new trials should focus also on the Android App (which was designed following the same device principles, but it has not been evaluated on the field yet).

It could also be useful and valuable to insert some specific didactical instruments (cognitive maps, vocal recording or reading, test customized formats, etc...) in order to extend the app with some tools helping dyslexic students both at school or home with the final aim of minimizing the individual difference in reading and learning for the student suffering diseases. In a context like the Italian school in which the number of students suffering DSA problems is increasing, social reading could be a way to compensate some learning diseases. At the time of writing we are collecting requirements in order to extend the app with tools for students suffering DSA.

\section{References}

1. http: //www . workinghomeguide.com/10763/huge-social-networkusers-growth-worldwide-by-2014-facebook-reinforce-dominance

2. http://blog.highbeambusiness.com/2011/08/pwc-report-digitalcontent-spurs-global-consumer-spending-growth/

3. http: //www.publishers.org/press/62/

4. Stephen, A.: P-Books vs. e-Books: Are there education issues? Multimedia and Internet @ Schools (November/ December 2010)

5. Scornavacca, Huff, Marshall: Mobile phones in the Classroom: If You Can't Beat Them, Join Them. Communications of the ACM 52(4) (April 2009)

6. Suthers, T.E.: Online Workspaces for Annotation and Discussion of Documents. In: ICCE International Conference on Computers in Education (2002)

7. Kim, Farzan, Brusilovsky: Social Navigation and Annotation for Electronic Books

8. Kiili, et al.: Working on Understanding During Collaborative Online Reaing. Journal of Literacy Research 44(4), 448-483 (2012) 